NASA Technical Memorandum 105841

\title{
Power System Monitoring and Source Control of the Space Station Freedom DC Power System Testbed
}

Greg L. Kimnach and Anastacio N. Baez Lewis Research Center

Cleveland, Ohio 


\title{
POWER SYSTEM MONITORING and SOURCE CONTROL of the SPACE STATION FREEDOM DC POWER SYSTEM TESTBED
}

\author{
Greg L. Kimnach and Anastacio N. Baez \\ National Aeronautics and Space Administration \\ Lewis Research Center \\ Cleveland, Ohlo 44135
}

\begin{abstract}
Unlike a terrestrial electric utility which can purchase power from a neighboring utility, the Space Station Freedom (SSF) has strictly limited energy resources; as a result, source control, system monitoring, system protection and load management are essential to the safe and efficient operation of the SSF Electric Power System (EPS). These functions are being evaluated in the DC Power Management and Distribution (PMAD) Testbed which NASA LeRC has developed at the Power System Facility (PSF) located in Cleveland, Ohio. The testbed is an ideal platform to develop, integrate, and verify power system monitoring and control algorithms.
\end{abstract}

State Estimation (SE) is a monitoring tool used extensively in terrestrial electric utilities to ensure safe power system operation. It uses redundant system information to calculate the actual state of the EPS, to isolate faulty sensors, to determine source operating points, to verify faults detected by subsidiary controllers, and to identify high impedance faults.

Source control and monitoring safeguard the power generation and storage subsystems and ensure that the power system operates within safe limits while satisfying user demands with minimal interruptions.

System monitoring functions, in coordination with hardware implemented schemes, provide for a complete fault protection system. The objective of this paper is to overview the development and integration of the state estimator and the source control algorithms.

\section{INTRODUCTION}

The NASA LeRC PMAD DC Testbed is a reduced scale representation of the SSF Electric PowerDistribution System (EPS). The testbed program objectives include supporting the identification of EPS operating scenarios, identifying potential technological risks, and evaluating potential control system design concepts. A complete description of the development and evaluation of the power system testbed to support the Space Station program is found in [Ref. 1].

\section{CONTROL SYSTEM OVERVIEW}

The DC Testbed control system is hierarchical, consists of eleven control nodes, and is based upon a Compaq 386/20e personal computer (PC) running ADA software. The monitoring and control functions, implemented in each node, ensure safe and efficient system operation. The hierarchical control scheme allows the control algorithms to execute quickly, and the ADA programming language is to provide a flexible platform for efficient control algorithm development and implementation.

Figure 1 is a block diagram of the DC PMAD Testbed highlighting the control- and distribution- system architectures. At the top of the hierarchical control scheme is the Operator Interface System (OIS) which is the main interface to the testbed operators and provides some of the functions that the Data Management System (DMS) will provide on the SSF. Among these functions are testbed software and hardware configuration and initialization, power system state monitoring, thermal system monitoring, graphical data display, command and control of all system components.

The Power Management Controller (PMC) is the EPS co-ordinator and its responsibilities, in general, are to coordinate the subsidiary controller functions and track overall primary distribution system performance. The PMC verifies and implements the Short Term Plan (STP), initializes the Primary Distribution System, and provides power system information to the OIS. The PMC communicates with the Subsidiary Level I and II controllers via an 802.4 token bus network [Ref. 2].

The Load Management Controller (LMC), also a Subsidiary Level I controller, co-ordinates the secondary and tertiary distribution system controllers. Its functions in the secondary distribution system are 
similar to the LMC functions, with the exception that it does not perform state estimation.

The Subsidiary Level II controllers are the Main Bus Controller (MBC) and the Photovoltaic Controller (PVC). The MBC monitors and controls the Main Bus Switching Unit (MBSU) and the DC-to-DC Converter the SSF-modules and payloads efforts, which are not the responsibility of the NASA LeRC. However, minimal monitoring and control functions are implemented to ensure the safe operation of the testbed hardware:
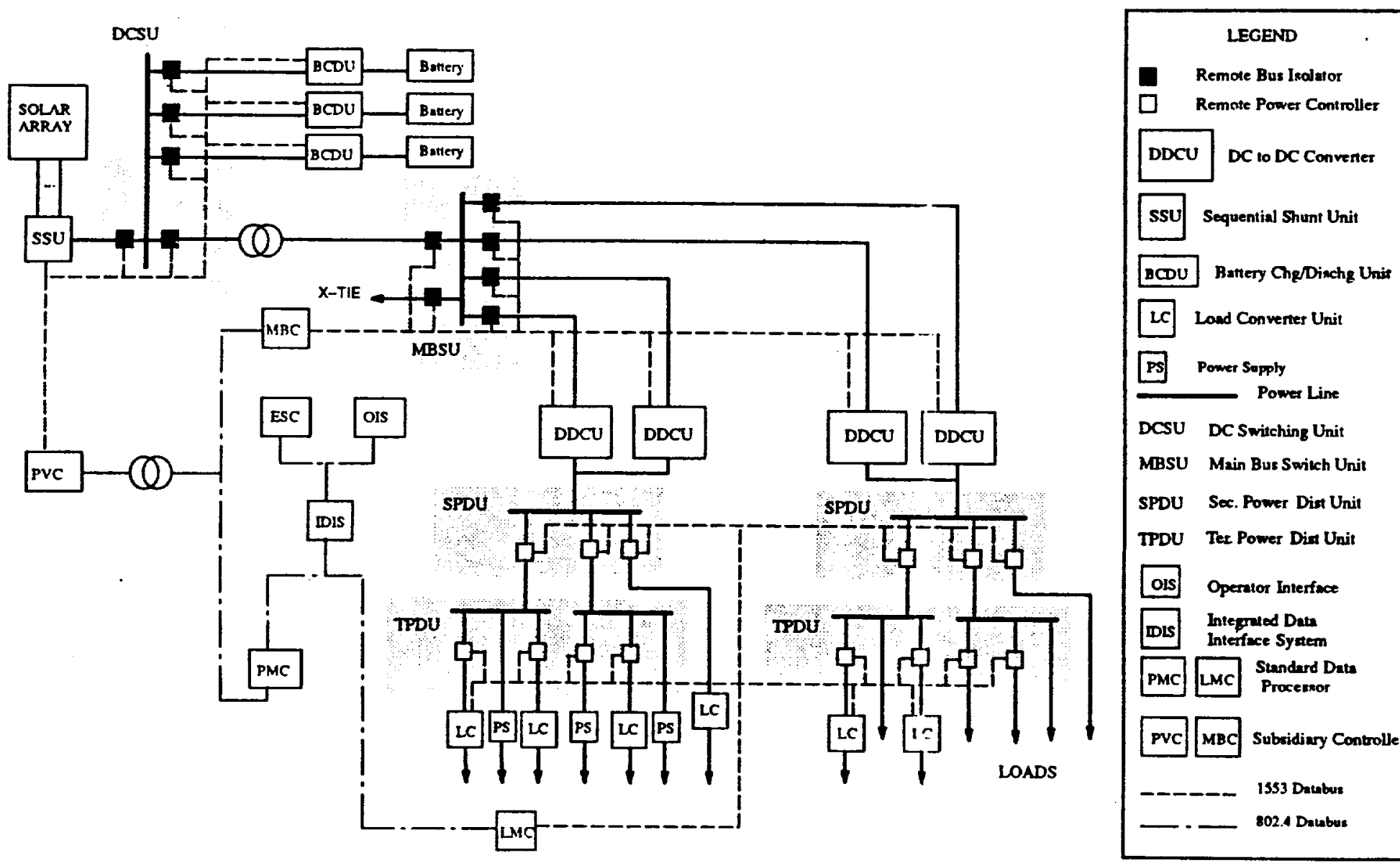

Power Management and Distribution Systems

Figure 1

Units (DDCUs); similarly, the PVC monitors and controls the Battery Charge/Discharge Units (BCDUs), the Direct Current Switching Unit (DCSU), and the Sequential Shunt Unit (SSU). These controllers communicate with the power components through a MIL-STD 1553B data bus.

The Secondary Distribution Controllers (SDC) and the Tertiary Power Controller (TPC) are also Subsidiary Level II controllers. The SDC and TPC monitor and control the secondary and tertiary distribution hardware, respectively. The design and development of these controllers, as well as that of the $\mathrm{LMC}$, fall within

\section{SYSTEM MONITORING}

The control system monitoring function is implemented by periodically sampling the power system components' data, smoothing it, analyzing it for acceptable system performance and preparing the necessary messages and commands for a given control node. The monitoring function requirements are to ensure the safe operation of the system; to track energy consumption and storage; to detect high-impedance faults; to verify lowimpedance faults detected by the subsidiary controllers, to verify and detect power interruptions, and constraint 
violations; to smooth data; to track the PDS efficiencies; and to provide the OIS with a smoothed EPS operating state. Figure 2 shows the interdependence of the algorithms discussed in this paper.

Some parameters that must be known accurately are source operating points (e.g., peak power point of solar array and remaining battery energy) and line currents: the former because efficiency and energy availability are of concern, and the latter because hardware protection is of concern.

At the local level data is digitally filtered with a thirdorder Butterworth filter, which smoothes the lowfrequency modulations. This filtered data is used to perform such local system protection functions as overand under-voltage detection, over-current detection (soft and hard), and hard-fault detection. Soft overcurrents are current values above the peak anticipated level as determined by a Load Flow Analysis (LFA) based on the peak demands as defined in the STP; hard over-currents are current values over the hardware ratings. Hard faults are those current levels which can be detected, by the local controllers usirg Kirchoff's Current Law and digitally filtered data; that is, current differences in excess of a sensors rating (5\%). Moreover, power-interrupt detection is accomplished using two consecutive, unfiltered data points. These schemes are the topic of [Ref. 2].

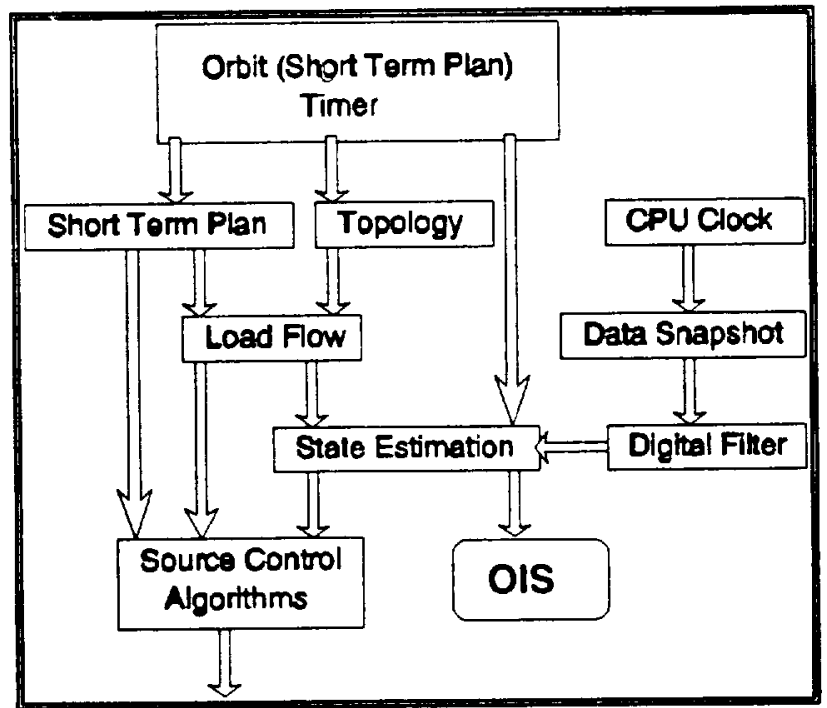

Algorithms Flow Diagram

Figure 2

The SE, running in the PMC, uses redundant, steadystate system measurements and sensor statistics to calculate the true operating state of the power distribution system and to identify unreliable sensorreadings. Because the SE gives the "true" operating state of the power-distribution system-unlike the Load Flow Analysis, which predicts the state based upon a priori knowledge of system topology, the reference-bus voltage, and loads--better control decisions can be made. SE is a weighted-least-squares estimate of the power system voltages given a known distribution topology. More detailed information about the SE is available in many references including [Ref. 4].

\section{STATE ESTIMATION INTEGRATION into the PMAD TEST BED's ADA SOFTWARE ENVIRONMENT}

SE, a Normai Mode algorithm [Ref. 2], is implemented in the PMC which, as the EPS co-ordinator, has access to all primary-distribution system measurements. The measurements sent to the PMC by the PVC and MBC controllers are current, voltage, and power readings at the SSU, DCSU, BCDUs, and MBSU.

The integration of the SE, implemented in ADA, is in progress and involves the creation of a "State Estimator Task" within the ADA runtime monitoring scheme. Ideally the SE task is to be run approximately every one second; however, due to the computational intensity of the algorithm and the complexity and overhead involved with the ADA environment, the task initially shall be called every ten seconds.

\section{SOURCE CONTROL}

Source control algorithms are required to efficiently and effectively service the user power requirements and to ensure the health of the energy storage system. All source control algorithms are dependent upon LFA results, filtered data, and Short Term Plan information, which defines the users' nominal and peak requirements over an entire orbit. The source control algorithms are used to initialize the source setpoints for the next demand period (each being one-minute) and to readjust the setpoints, as necessary, during a demand period. The focus of this section is the initialization of the BCDUs and energy management of the batteries.

Before describing the BCDU-control algorithms, some basic battery and BCDU concepts are given.

\section{BCDU and BATTERY CONCEPTS}

The BCDUs are bi-directional-power converters which, depending upon the BCDU setpoints and primary distribution bus voltages, regulate the battery-charge current, the bus-discharge current, or the bus voltage. The BCDUs have five setpoints which are adjusted by 
the source-control algorithms, are shown in Figure 2, and are as follows:

(1) The Bus-Voltage Setpoint (BVS) is a reference for other modes of operation. These modes are the Charge-Reduction and Discharge-Reduction Mode.

(2) The Charge-Current Setpoint (CCS) is a charge-current limit into the batteries. The BCDUs will regulate the current at this value for bus voltages above a given threshold. Below this threshold--equal to the DCSU-bus voltage predicted by the LFA of the peak demands (P-LFA), but above the BVS-the BCDUs enter the Charge Reduction Mode and, thus, allow the SSU to regulate the system voltage.

(3) Deadband Setpoint (DbS) (or Skew Voltage Setpoint) keeps the BCDU from oscillating between charge and discharge modes.

(4) Discharge-Current Gain Setpoint (DGS) determines the degree to which the BCDU will regulate the bus voltage ( $160 \mathrm{~V}$ side).

(5) Discharge-Current Setpoint (DCS) which limits the current injection into the primary distribution system.

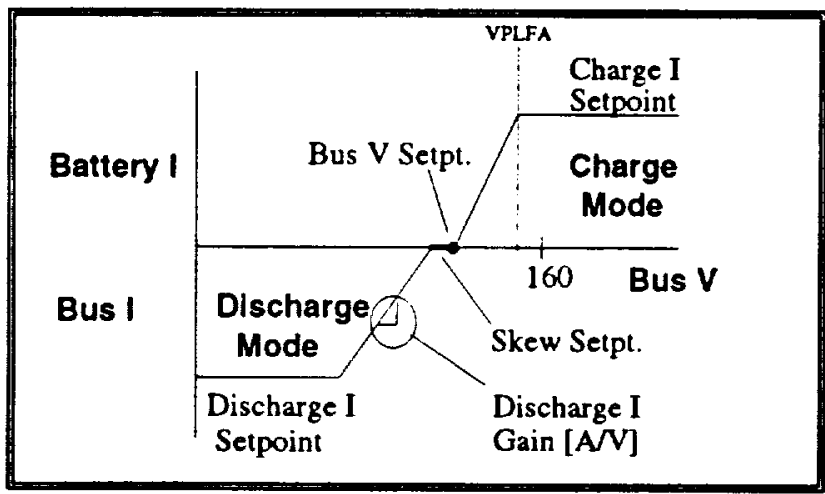

Current-vs-Bus-Voltage during Insolation Figure 3

For further information about BCDU operation see [Ref. 4]. The batteries and battery simulators used in the PMAD testbed have 80AHr Capacities (C) and the following constraints are important for battery cycling:

(1) The batteries must reach $100 \%$ State of Charge (SoC) by the onset of eclipse. (This mandates an accurate means of monitoring the batteries.) Battery capacity is a function of many parameters (e.g., temperature, cycles, and discharge current) and the residual capacity, expressed as SoC, is most readily calculated by integrating the battery current. Two forms of the SoC calculation are as follows: (a) While charging, some of the energy does not reverse the chemical reaction of the battery, but is lost as heat; this inefficiency in recharging a battery is accounted for by a recharge ratio (r.r.). As a result, Equation 1 is used to calculate the residual capacity of the battery.

$$
\operatorname{SoC}(\mathbf{k})[\%]=\operatorname{SoC}(\mathbf{k - 1})[\%]+100 \frac{\text { Inilered (k) }[\mathrm{A}]^{*} \Delta \mathrm{T}[\mathrm{sec}]}{\text { r.r. }{ }^{*} \text { Capacity[Asec] }}
$$

\section{SoC Calculation: Charge Mode Equation 1}

(b) While discharging, the effective capacity of a battery decreases with increased current draw, thus the capacity at different operating levels must be accounted for and is accomplished according to Equation 2.

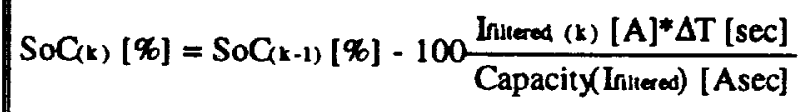

\section{SoC Calculation: Discharge Mode Equation 2}

A plot of a typical discharge-time-vs-current of batteries is shown in Figure 3. Note that the capacity at a given current level is simply found by multiplying the current [A] by the time[Hrs].

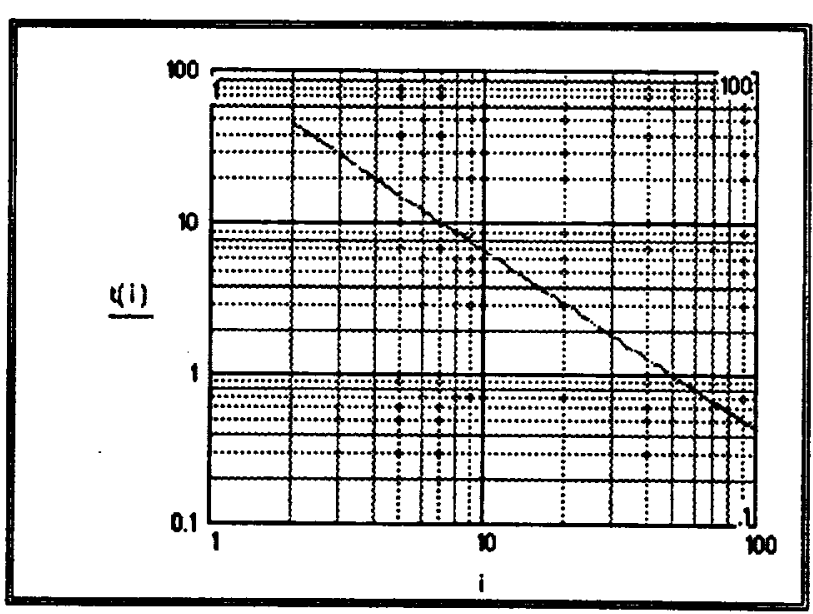

\section{Discharge Time (Hrs)-vs-Current (Amps) Figure 4}

(2) Limit the charge current to the maximum allowable. If possible, use a high initial charge current value and decrease as the battery approaches full SoC.

(3) Control charge current so that heat buildup is minimized. 
(4) As the battery SoC approaches $100 \%$, limit the current to a "trickle" of $0.01 \mathrm{C}$, for example.

It should be noted that the charge-profile for the batteries is not within the scope of this paper and is assumed to be given a priori. However, modifications can, and are, made to the profile as needed.

\section{BCDU INITIALIZATION during INSOLATION}

BCDU initialization, using load-flow analysis results and battery measurements, is required to ensure that the batteries are fully charged by the onset of eclipse. The initialization procedure also allows for possible power augmentation of the SSU in the presence of heavy loading. Note, however, that the fault identification algorithms shall keep the BCDUs from discharging the batteries into a fault.)

Although the programmatic constraints presently preclude discharging the batteries during insolation, the advantages of allowing such operation, combined with the simple algorithm to determine discharge setpoints, which still fulfill the $100 \%$ SoC requirement at the onset of eclipse, justify the possibility of discharging during insolation. If discharging during insolation remains unallowed, then these algorithms can either be modified or the maximum-allowable discharge-current values set to zero within the database. That is, the algorithms are flexible enough to implement either mode.

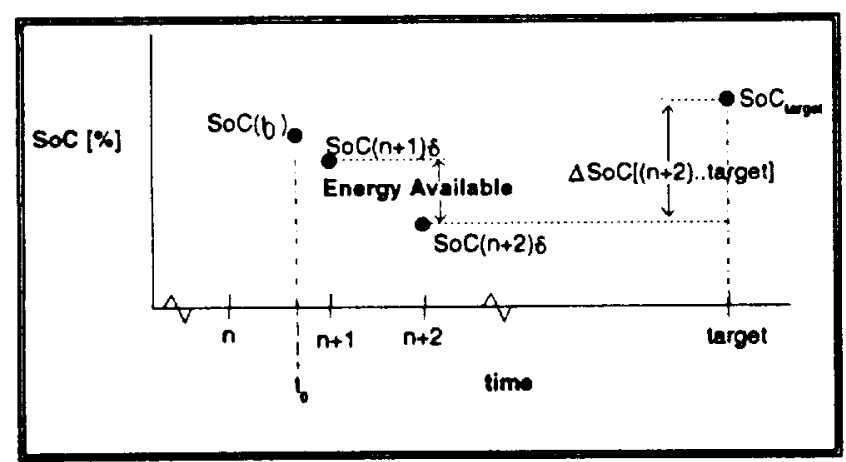

Discharge Capacity Available during $(n+1) T$ Time Line 1

The terms in Equation 3 include:

$$
\text { SoC }(n+1) T-\delta \text { is the minimum expected SoC }
$$
of a battery at the end of the present demand period. It is calculated at $t_{b}$ and assumes that the battery shall continue to operate in its present mode. This term is abbreviated as $\mathrm{SoC}^{0}{ }_{\text {min }}$.

$$
\triangle S o C(n+1) T \text { is the amount of energy that a }
$$
battery can supply during the next period. It is constrained to not fall below the minimum SoC (i.e., $65 \%$ ) and still ensure that the battery reach its target value based on $\Delta S o C_{[(n+2) \ldots t u d]}$. This is refered to as "Energy Available" in Time Line 1.

$\Delta S o G_{(n+2) . . m e r]}$ is the amount of energy that can be guaranteed to be stored from the end of the next demand period to the "target" time.

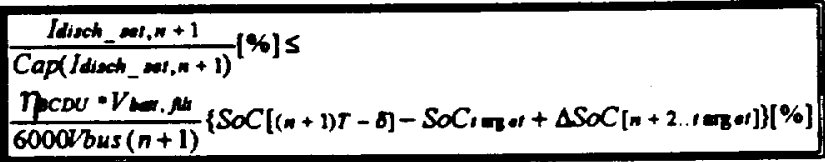

$$
\begin{aligned}
& \text { Discharge-Current Setpoint( } n+1) \text { Calculation } \\
& \text { Equation } 3
\end{aligned}
$$

The initialization process begins by checking the shortterm plan for the minimum charge-current available to charge the batteries in the period $[(n+2)$..target time] and setting the charge-current setpoint values according to this value and their relative SoCs. Having fixed the charge-current setpoint values in the STP, the maximum discharge current--for batteries which have reached a minimum SoC of $85 \%$, for example--is determined for the next demand period according to Equation 3. Because the capacity of a battery is a function of its discharge-current magnitude, the current is that value which satisfies the ratio in Equation 3, and is found in a look-up table. Furthermore, this equation is based on the constraint that the STP is implemented without modification to the user demands and the batteries reach a "target" SoC at the appropriate time. Having determined the discharge-current limit upon the batteries, the remaining BCDU setpoints are readily determined.

The bus voltage setpoint values are a function of the DCSU-voltage predicted by the a load-flow analysis run on the peak demands: this is the voltage at which the BCDUs are to enter the Charge Reduction Mode-and from Figure 2 are seen to be:

$$
\begin{gathered}
\text { Bus_Voltage_Setpoint }=\frac{- \text { Charge_Current_Setpoint }_{\text {Discharge_Current_Gain }}+V D C S U, P L F A}{\text { Bus Voltage Setpoint Calculation }} \\
\text { Equation } 4
\end{gathered}
$$

The deadband values are set to the maximum of one volt and the discharge gains are set to the maximum slope of $60[\mathrm{~A} / \mathrm{V}]$ to better regulate the system during the heavy-loading (which should be infrequent).

\section{BCDU INITIALIZATION DURING ECLIPSE}

During eclipse the batteries are the sole sources for users' powver requirements. As such, the BCDUs must 
provide nominal and peaking requirements and must regulate the system voltage. Further, in conjunction with load-control algorithms and the STP, it regulates the energy supplied by the batteries so that energy is available throughout eclipse. Ideally, the batteries shall supply energy in a balanced manner. Finally, the assumptions are made that the discharge-current setpoint for the next and subsequent periods is that nominal value which would reduce a given battery's $\mathrm{SoC}$ to its minimum value by the end of eclipse, and that batteries have identical SoCs (i.e., 100\%) at the onset of eclipse.

Before the initialization of the BCDU setpoints is performed, the SoC of each battery is calculated according to Equation 2. The battery having the highest SoC is referred to as the master and the other two batteries are the slaves, moreover, the associated BCDUs are referred to as master and slaves, also. These rankings of the SoCs are required to appropriately determine the discharge-current setpoints of the BCDUs.

Because the BCDU/battery pairs do not operate identically, the SoCs shall drift apart and must be "rebalanced." The Discharge-current setpoints shall result in a rebalancing of the SoCs. Assuming that the BCDU-bus voltages are each equal to the desired DCSU voltage of $160 \mathrm{~V}$, and by substituting and reflecting to the "battery side" the following constraint

$$
\text { IBCDU,mas = IDCSU,som -IBCDU,slave1 - IBCDU, slave2 }
$$

into the two battery equations:

$$
\begin{aligned}
& S \alpha C_{\text {min ,slaw 1 }}^{0}-\frac{6000 I_{\text {bar , slave } 1}(n+1)}{\operatorname{Cap}\left(I_{\text {barl, slawe } 1}(n+1)\right)}= \\
& S \alpha C_{\text {master }}^{0}-\frac{6000 I_{\text {batt,master }}(n+1)}{C a p\left(I_{\text {ban, marter }}(n+1)\right)} \\
& S \propto C_{\min , \text { slawe } 2}^{0}-\frac{6000 I_{\text {batt, slave } 2}(n+1)}{C a p\left(I_{\text {batt , slave } 2}(n+1)\right)}= \\
& S \alpha C_{\text {master }}^{0}-\frac{6000 I_{\text {batt,master }}(n+1)}{\operatorname{Cap}\left(I_{\text {batt,master }}(n+1)\right)}
\end{aligned}
$$

Simultaneous Equations to Rebalance State of Charge Equations 5 a and $5 b$

gives the required simultaneous equations to determine the discharge-current values to rebalance the batteries. Again, the discharge-current values are found in a look-up table of values.

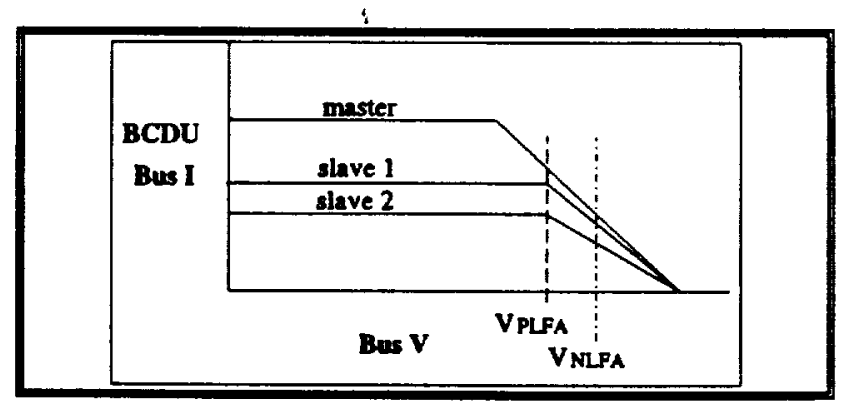

\section{BCDU Setpoints during Eclipse \\ Figure 5}

The discharge-current gain for the master is the slope calculated by using the difference between the minimum-desired DCSU-bus voltage and the nominal voltage as the "run"; the difference between the nominal and peak currents is the "rise." The busvoltage setpoint is found by determining the intercept of a linear equation.

\section{SOURCE CONTROL INTEGRATION}

The source control integration process is similar to the state estimation. Source control algorithms are implemented as Ada tasks and are resident in the PMC. The source control software will eventually reside in the PVC.

\section{CONCLUSIONS}

Monitoring and control algorithms are being developed to enhance the PMAD DC testbed power system overall operating efficiency. Source control algorithms provide the benefits of an increased in energy available to the users while maintaining stable system operation. State estimation techniques are being used to detect highimpedance fault in the power distribution system and to verify the results of low level fault detection algorithms.

\section{ACKNOWLEDGMENTS}

The authors would like to thank Dave Gantose and Kim Ludwig of Sverdrup Technologies, Inc. and Jim Withrow of LeRC for their efforts in integrating the algorithms into the ADA-runtime environment. Also, Don Fong of LeRC for his assistance during testing.

\section{REFERENCES}

[1] J.F. Soeder and RJ. Frye (NASA LeRC, Cleveland, $\mathrm{OH}$ ), "Overiew and Evolution of the LeRC PMAD DC Testbed" IECEC 1992 Proceedings.

[2] A.N. Baez and G.L. Kimnach (NASA LeRC, Cleveland, $\mathrm{OH}$ ), "Description of the Control System 
Design for the SSF PMAD DC Testbed" IECEC 1991 Proceedings, pp. 334-339.

[3] A.N. Baez, M. Mackin, and T. Wright (NASA LeRC, Cleveland, OH), "Description of the SSF DC PMAD Testbed Control System Data Aquisition Function" IECEC 1992 Proceedings.

[4] G. L. Kusic, Computer Aided Power Systems Analysis. Prentice Hall, 1986. Pp. 347-381.

[5] R.M. Button (NASA LeRC, Cleveland, $\mathrm{OH}$ ), "Development and Testing of a Source Subsystem for the Supporting Development PMAD DC Testbed" IECEC 1991 Proceedings, pp. 365-367. 
Public reporting burden for this collection of information is estunaled to average 1 hour per response, including the time for reviewing instructions, searching existing data sources. gathering and maintaining the date needed. and completing and reviewing the collection of information. Send comments regarding this burden estimate or any other aspect of this collection of information, including suggestions for reducing this burden, to Washington Headquarters Services. Directorate for inforrpation Operations and Reports, 1215 Jefferson Davis Highway. Suite 1204. Arlington, VA 22202-4302, and to the Office of Management and Budget, Papenwork Peduction Project (0704-0188). Washington, DC 20503.

\begin{tabular}{l|l} 
1. AGENCY USE ONLY (Lave blank) & 2. REPORT DATE \\
August 1992
\end{tabular}

\section{TILE AND SUBTITLE}

August 1992
3. REPOAT TYPE AND DATES COVERED

Technical Memorandum

Power System Monitoring and Source Control of the Space Station

Freedom DC Power System Testbed

6. AUTHOR(S)

WU $-474-42-10$

Greg L. Kimnach and Anastacio N. Baez

7. PERFORMING ORGANIZATION NAME(S) AND ADDRESS(ES)

8. Pefforming organization REPORT NUMBER

National Aeronautics and Space Administration

Lewis Research Center

Cleveland, Ohio 44135-3191

E-7284

9. SPONSORING/MONITORING AGENCY NAMES(S) AND ADDRESS(ES)

10. SPONSORING/MONITORING AGENCY REPORT NUMBER

National Aeronautics and Space Administration

Washington, D.C. 20546-0001

NASA TM-105841

\section{SUPPLEMENTARY NOTES}

Prepared for the 27th Intersociety Energy Conversion Engineering Conference cosponsored by the SAE, ACS, AIAA, ASME, IEEE, AIChE, and ANS, San Diego, California, August 3-7, 1992. Greg L. Kimnach and Anastacio N. Baez, NASA Lewis Research Center, Cleveland, Ohio. Responsible person, Greg L. Kimnach, (216) 433-8323.

\begin{tabular}{l|l} 
12a. DISTAIBUTION/AVAILABILITY STATEMENT & 12b. DISTRIBUTION CODE
\end{tabular}

Unclassified - Unlimited

Subject Categories 20 and 33

13. ABSTRACT (Maximum 200 words)

Unlike a terrestrial electric utility which can purchase power from a neighboring utility, the Space Station Freedom (SSF) has strictly limited energy resources; as a result, source control, system monitoring, system protection and load management are essential to the safe and efficient operation of the SSF Electric Power System (EPS). These functions are being evaluated in the DC Power Management and Distribution (PMAD) Testbed which NASA LeRC has developed at the Power System Facility (PSF) located in Cleveland, Ohio. The testbed is an ideal platform to develop, integrate, and verify power system monitoring and control algorithms. State Estimation (SE) is a monitoring tool used extensively in terrestrial electric utilities to ensure safe power system operation. It uses redundant system information to calculate the actual state of the EPS, to isolate faulty sensors, to determine source operating points, to verify faults detected by subsidiary controllers, and to identify high impedance faults. Source control and monitoring safeguard the power generation and storage subsystems and ensure that the power system operates within safe limits while satisfying user demands with minimal interruptions. System monitoring functions, in coordination with hardware implemented schemes, provide for a complete fault protection system. The objective of this paper is to overview the development and integration of the state estimator and the source control algorithms.

14. SUBJECT TERMS
Control systems; Space power systems; Power management

\begin{tabular}{|l|c|}
\hline & $\begin{array}{c}\text { 15. NUMBER OF PAGES } \\
8\end{array}$ \\
\cline { 2 - 2 } & $\begin{array}{c}\text { 16. PAICE CODE } \\
\text { AO2 }\end{array}$ \\
\hline $\begin{array}{l}\text { 19. SECURTY CLASSIFICATION } \\
\text { OF ABSTRACT } \\
\text { Unclassified }\end{array}$ & $\begin{array}{l}\text { 20. LIMITATION OF ABSTRACT } \\
\end{array}$
\end{tabular}

18. SECURTY CLASSIFICATION OF THIS PAGE Unclassified 